\title{
Nonadiabatic noncyclic geometric quantum computation in Rydberg atoms
}

\author{
Bao-Jie Liu $\odot,{ }^{1,2}$ Shi-Lei Su $\odot,{ }^{3, *}$ and Man-Hong Yung ${ }^{2,4,5, \dagger}$ \\ ${ }^{1}$ Department of Physics, Harbin Institute of Technology, Harbin 150001, China \\ ${ }^{2}$ Institute for Quantum Science and Engineering, and Department of Physics, Southern University of Science and Technology, \\ Shenzhen 518055, China \\ ${ }^{3}$ School of Physics, Zhengzhou University, Zhengzhou 450001, China \\ ${ }^{4}$ Guangdong Provincial Key Laboratory of Quantum Science and Engineering, Southern University of Science and Technology, \\ Shenzhen 518055, China \\ ${ }^{5}$ Shenzhen Key Laboratory of Quantum Science and Engineering, Shenzhen 518055, China
}

(Received 23 June 2020; accepted 6 October 2020; published 23 October 2020)

\begin{abstract}
Nonadiabatic geometric quantum computation (NGQC) has been developed to realize fast and robust geometric gate. However, the conventional NGQC is that all of the gates are performed with exactly the same amount of time, whether the geometric rotation angle is large or small, due to the limitation of cyclic condition. Here, we propose an unconventional scheme, called nonadiabatic noncyclic geometric quantum computation (NNGQC), that arbitrary single- and two-qubit geometric gate can be constructed via noncyclic non-Abelian geometric phase. Consequently, this scheme makes it possible to accelerate the implemented geometric gates against the effects from the environmental decoherence. Furthermore, this extensible scheme can be applied in various quantum platforms, such as superconducting qubit and Rydberg atoms. Specifically, for single-qubit gate, we make simulations with practical parameters in neutral atom system to show the robustness of NNGQC and also compare with NGQC using the recent experimental parameters to show that the NNGQC can significantly suppress the decoherence error. In addition, we also demonstrate that nontrivial two-qubit geometric gate can be realized via unconventional Rydberg blockade regime within current experimental technologies. Therefore, our scheme provides a promising way for fast and robust neutral-atom-based quantum computation.
\end{abstract}

DOI: 10.1103/PhysRevResearch.2.043130

\section{INTRODUCTION}

Neutral atoms that interact via dipole-dipole interactions have became a potential platform for quantum computation $[1,2]$. Rydberg atoms are one kind of neutral atoms that are excited to high-lying Rydberg states [3], which would exhibit strong Rydberg dipole-dipole interaction when the interatomic distance is not very large. And the RydbergRydberg interaction (RRI) have been studied for construction of quantum logic gates [4-6]. By using microwave transitions, single-qubit Rabi oscillation of neutral atoms have been well studied experimentally [7]. Besides, high fidelity single-qubit quantum logic gates [8-11] and quantum controls [12] have also been demonstrated in neutral atoms. Through the laserinduced transitions from ground state to Rydberg state, many two- and multiple-qubit gates in neutral atom based on RRI have also been demonstrated in experiments [13-21]. These experimental studies show the high-fidelity of single-qubit

\footnotetext{
*slsu@zzu.edu.cn

†yung@sustech.edu.cn

Published by the American Physical Society under the terms of the Creative Commons Attribution 4.0 International license. Further distribution of this work must maintain attribution to the author(s) and the published article's title, journal citation, and DOI.
}

gates and also show how to improve the fidelity of two-qubit gates step by step. On that basis, if one can design single- and two-qubit quantum logic gates that are more robust to systematic fluctuation error and decoherence, it will be beneficial to realize quantum computation in neutral atoms.

Geometric quantum logic gates $[22,23]$ based on adiabatic or nonadiabatic geometric phase [24-27], which depends only on the global properties of the evolution paths, provides us the possibility for robust quantum computation [28-34]. In contrast to the earlier adiabatic-process-based geometric quantum computation [35-38], nonadiabatic geometric quantum computation (NGQC) and nonadiabatic holonomic quantum computation (NHQC) based on Abelian [39-46] and non-Abelian geometirc phases [47-56] in two- and threelevel system, respectively, can intrinsically protect against environment-induced decoherence, since the the construction times of geometric quantum gates is reduced. The nonadiabatic geometric gates of NGQC and NHQC have been experimentally demonstrated in many systems including superconducting qubit [57-61], NMR [62-65], NV center in diamond [66-69]. However, there are many theoretical proposals to apply geometric quantum computation [70-73] and NGQC [42,74-77] in Rydberg atom platform. To further speed up the NGQC scheme, NGQC is incorporated with the time-optimal technology to realize the geometric gate with the minimum gate under the framework of cyclic evolution [78-80]. However, both NGQC and time-optimal NGQC 
should satisfy the cyclic condition, which leads to neutralatom-based quantum logic gates being more sensitive to decay and dephasing errors compared to the conventional dynamical counterparts [8-10]. Particularly, the evolution time of NGQC should be exactly the same for all quantum logic gates no matter the geometric rotation angle is large or small.

Here, we propose a new scheme, nonadiabatic noncyclic geometric quantum computation (NNGQC), that all of singlequbit geometric gate and nontrivial two-qubit can be realized via noncyclic non-Abelian geometric phase in a Rydberg system. Comparing with the conventional Rydberg blockade [4-6], we consider RRI-induced blockade process seriously by second-order dynamics, which may be more accurate since we do not discard the stark shifts relevant to the "blockade". More importantly, our scheme can further reduce the geometric gate time of NGQC [42-46] without the limitation of cyclic condition. Specifically, we found that the certain gate time of NNGQC can be reduced by half compared with NGQC by choosing proper control parameter. The numerically thorough analysis show that, under the same experimental conditions, the decay and dephasing error caused by the environmental noise can significantly be suppressed via our NNGQC rather than the conventional NGQC. Comparing with recently noncyclic schemes $[81,82]$, (i) our scheme only needs to adjust the amplitude and phase of microwave field without complicated pulse sequences in a resonant two-level system; (ii) the gate speed of our scheme is faster than Ref. [82] with the same Rabi frequency. Furthermore, our NNGQC can also be conveniently applied to other physical platforms such as superconducting qubits [57] and nitrogen-vacancy centers $[66,67]$.

\section{GENERAL MODEL}

Here, we consider a ${ }^{133} \mathrm{Cs}$ with magnetically insensitive "clock" states encoding $|0\rangle \equiv\left|6 S_{1 / 2}, F=3, m_{F}=0\right\rangle$ and $|1\rangle \equiv\left|6 S_{1 / 2}, F=4, m_{F}=0\right\rangle$ [83], which is resonantly driven by a microwave pulse to realize the transitions of $|0\rangle \leftrightarrow|1\rangle$ with the Rabi frequency $\Omega(t)$ and phase $\varphi(t)$, as shown in Fig. 1(a). The Hamiltonian for this system is (here and after $\hbar \equiv 1) H(t)=\frac{\omega_{0}}{2}(|1\rangle\langle 1|-| 0\rangle\langle 0|)+$ $\Omega(t)\left\{\cos \left[\omega_{0} t+\phi(t)\right]|1\rangle\langle 0|+\right.$ H.c. $\}$, where $\omega_{0}$ denotes the corresponding transition energy. In the rotating wave approximation and the interaction frame, the system is given by a time-dependent Hamiltonian,

$$
H=\frac{1}{2}\left(\begin{array}{cc}
0 & \Omega(t) e^{-i \varphi(t)} \\
\Omega(t) e^{i \varphi(t)} & 0
\end{array}\right) .
$$

For a pair of basis vectors $\left\{\left|\psi_{1}(t)\right\rangle,\left|\psi_{2}(t)\right\rangle\right\}$ following the Schrödinger's equation as $\left|\psi_{1,2}(t)\right\rangle=\mathcal{T} e^{-i \int_{0}^{t} H\left(t^{\prime}\right) d t^{\prime}}\left|\psi_{1,2}(0)\right\rangle$, the time-evolution operator can be given by $U(t, 0)=$ $\mathcal{T} e^{-i \int_{0}^{t} H\left(t^{\prime}\right) d t^{\prime}}=\left|\psi_{1}(t)\right\rangle\left\langle\psi_{1}(0)|+| \psi_{2}(t)\right\rangle\left\langle\psi_{2}(0)\right|$. Now, we take a set of auxiliary states $\left|\phi_{1}(t)\right\rangle=\left(\cos \frac{\chi}{2} \mathrm{e}^{-\mathrm{i} \frac{\eta}{2}}, \sin \frac{\chi}{2} \mathrm{e}^{\mathrm{i} \frac{\eta}{2}}\right)^{\mathrm{T}}$ and $\left|\phi_{2}(t)\right\rangle=\left(\sin \frac{\chi}{2} \mathrm{e}^{-\mathrm{i} \frac{\eta}{2}},-\cos \frac{\chi}{2} \mathrm{e}^{\mathrm{i} \frac{\eta}{2}}\right)^{\mathrm{T}}$, with the boundary conditions $\left|\phi_{m}(0)\right\rangle=\left|\psi_{m}(0)\right\rangle$ at time $t=0$. In this way, $\left|\psi_{m}(t)\right\rangle$ can be expressed $\left|\psi_{m}(t)\right\rangle=\sum_{l} C_{l m}(t)\left|\phi_{l}(t)\right\rangle$, and the time-evolution operator becomes $U(t, 0)=$ $\sum_{l, m} C_{l m}(t)\left|\phi_{m}(t)\right\rangle\left\langle\phi_{m}(0)\right|$. Using the Schrödinger's equation, we obtain the final time evolution operator

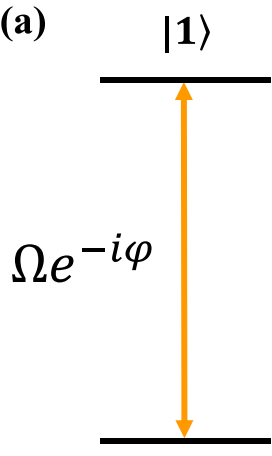

$|\mathbf{0}\rangle$ (b)

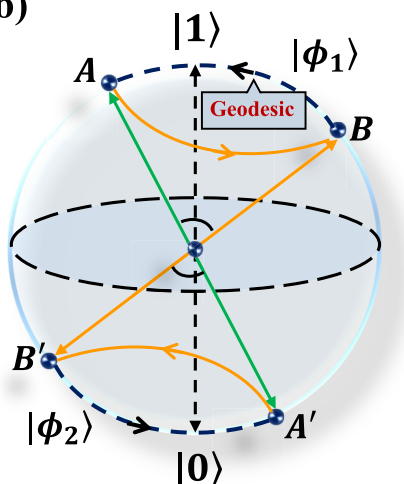

FIG. 1. The illustration of our proposed implementation. (a) The two-level energy structure is resonantly driven by a microwave pulse to realize the transitions of $|0\rangle \leftrightarrow|1\rangle$ with the Rabi frequency $\Omega(t)$ and phase $\varphi(t)$. (b) Conceptual explanation for noncyclic geometric quantum operation. Noncyclic gemetirc phase is given by half the solid angle enclosed by the trajectory $A B\left(A^{\prime} B^{\prime}\right)$ and the geodesic $B A\left(B^{\prime} A^{\prime}\right)$ connecting the initial and final points.

$U(\tau, 0)=\sum_{l, m=1}^{2}\left(\mathbf{T e}^{\mathrm{i} \int_{0}^{\tau}[\mathbf{A}(t)+\mathbf{K}(t)] \mathrm{d} t}\right)_{l m}\left|\phi_{l}(\tau)\right\rangle\left\langle\phi_{m}(0)\right|, \quad$ with $\mathbf{A}_{l m} \equiv i\left\langle\phi_{l}(t)|(d / d t)| \phi_{m}(t)\right\rangle \quad$ being the matrix-valued connection one-form and $\quad \mathbf{K}_{l m}(t) \equiv-\left\langle\phi_{l}(t)|H(t)| \phi_{m}(t)\right\rangle$ being dynamical part. If a special auxiliary state is chosen to make dynamical part varnished, a noncyclic holonomy matrix can be obtained [84].

To realize a noncyclic geometric gate, we choose the auxiliary state $\left|\phi_{m}(t)\right\rangle$ to be proportional to the dynamical states $\left|\psi_{m}(t)\right\rangle$, which satisfies the von Neumann equation [49]: $\frac{d}{d t} \Pi_{m}(t)=-i\left[H(t), \Pi_{m}(t)\right]$, where $\Pi_{m}(t) \equiv\left|\phi_{m}(t)\right\rangle\left\langle\phi_{m}(t)\right|$ denotes the projector of the auxiliary basis. Explicitly, we found that they are governed by the following coupled differential equations:

$$
\Omega(t)=\frac{\dot{\chi}}{\sin (\varphi-\eta)}, \quad \varphi(t)=\eta-\arctan \left(\frac{\dot{\chi}}{\dot{\eta} \tan \chi}\right) .
$$

In this way, the time-evolution operator becomes

$$
U(\tau, 0)=e^{i \gamma}\left|\phi_{1}(\tau)\right\rangle\left\langle\phi_{1}(0)\left|+e^{-i \gamma}\right| \phi_{2}(\tau)\right\rangle\left\langle\phi_{2}(0)\right|,
$$

where $\quad \gamma(\tau)=\int_{0}^{\tau}\left(\mathbf{A}_{11}+\mathbf{K}_{11}\right) d t=-\int_{0}^{\tau}\left(\mathbf{A}_{22}+\mathbf{K}_{22}\right) d t=$ $\int_{0}^{\tau} \frac{\dot{\eta}}{2 \cos \chi} d t$ denotes global phase including the diagonal geometric phase $\gamma_{g}=\int_{0}^{\tau} \mathbf{A}_{11} d t=\int_{0}^{\tau} \frac{1}{2} \dot{\eta} \cos \chi d t$ and diagonal dynamical phase $\gamma_{d}=\int_{0}^{\tau} \mathbf{K}_{11} d t=$ $-\int_{0}^{\tau} \Omega \cos \chi \cos (\varphi-\eta) d t$. To make evolution gate in Eq. (3) purely geometric, we set $\varphi-\eta=\pi / 2$ for erasing the diagonal dynamical phase. Therefore, the diagonal geometric phase $\gamma=\int_{\eta(0)}^{\eta(\tau)} \int_{\chi(0)}^{\chi(\tau)} \frac{1}{2} \sin \chi d \chi d \eta=\Omega_{\text {angle }} / 2$ is given by half the solid angle enclosed by the trajectory and the geodesic connecting the initial and final points, as shown in Fig. 1(b). Finally, the evolution operator in the basis $\{|0\rangle,|1\rangle\}$ is found to be

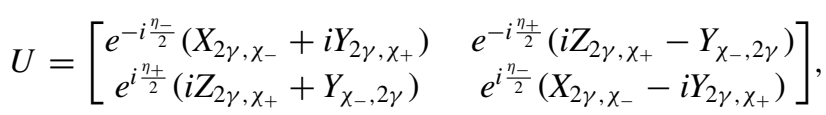


where $\quad X_{a, b} \equiv \cos \frac{a}{2} \cos \frac{b}{2}, \quad Y_{a, b} \equiv \sin \frac{a}{2} \cos \frac{b}{2}, \quad Z_{a, b} \equiv$ $\sin \frac{a}{2} \sin \frac{b}{2}, \quad \chi_{ \pm}=\chi(\tau) \pm \chi(0), \quad$ and $\quad \eta_{ \pm}=\eta(\tau) \pm \eta(0)$. The initial values of the auxiliary variables $\eta$ and $\chi$ can be determined by the target geometric gates.

To illustrate the geometric rotation, $U$ is conveniently represented (ignore a global phase) as

$$
U(\theta, \alpha, \beta)=Z_{\beta} X_{\theta} Z_{\alpha},
$$

where $\theta \equiv \sin ^{-1}\left(\sqrt{\left.Z_{2 \gamma, \chi_{+}}^{2}+Y_{\chi_{-}, 2 \gamma}^{2}\right)}, \alpha \equiv-\tan ^{-1}\left(\frac{Y_{2 \gamma, \chi_{+}}}{X_{2 \gamma, \chi_{-}}}\right)-\right.$ $\tan ^{-1}\left(\frac{Z_{2 \gamma, x_{+}}}{Y_{x_{-}, 2 \gamma}}\right)+\frac{\eta_{-}-\eta_{+}-\pi}{2}, \quad$ and $\quad \beta \equiv-\tan ^{-1}\left(\frac{Y_{2 \gamma, x_{+}}}{X_{2 \gamma_{-}, x_{-}}}\right)+$ $\tan ^{-1}\left(\frac{Z_{2 \gamma, X_{+}}}{Y_{X_{-}, 2 \gamma}}\right)+\frac{\eta_{-}+\eta_{+}+\pi}{2}$ are rotation of angles around the $X$ and $Z$ axis of the Bloch sphere, respectively. Any single-qubit $S U$ (2) operation can be realized with $U(\theta, \alpha, \beta)$ by choosing the geometric phase $\gamma$, the initial and final value $\eta_{ \pm}$and $\chi_{ \pm}$. For example, we can realize the noncyclic geometric $U_{1}=U(\pi / 2,-\pi / 2,0)$ and $U_{2}=U(\pi / 2, \pi / 2, \pi / 2)$ (Hardmard gate) by setting the parameters as $\left\{\gamma=\frac{\pi}{4}, \chi_{+}=0, \chi_{-}=\pi, \eta_{ \pm}=\mp \frac{\pi}{2}\right\}$, and $\left\{\gamma=\frac{\pi}{4}, \chi_{+}=-\frac{3 \pi}{2}, \chi_{-}=\frac{\pi}{2}, \eta_{ \pm}=\mp \frac{\pi}{2}\right\}, \quad$ respectively. More specifically, we note that Eq. (4) is the $Z$-rotation gate $Z_{\left(-\eta_{-}\right)}=\exp \left(i \frac{\eta_{-}}{2} Z\right)$ when $\gamma=\pi, \chi_{-}=0$ and is the $X$-rotation gate $X_{\chi_{-}}=\exp \left(i \frac{\chi_{-}}{2} X\right)$ when $\gamma=\pi, \eta_{-}=0$ and $\eta_{+}=-\pi$.

To further understand the scheme of our NNGQC, we found that the nondiagonal parts of $\mathbf{A}$ and $\mathbf{K}$ satisfy the relations of unconventional quantum holonomy [50,85] as $\int_{0}^{\tau} \mathbf{A}_{k m} d t / \int_{0}^{\tau} \mathbf{K}_{k m} d t=-1$ for $k \neq m$, where $\mathbf{A}_{k m}(t)=$ $\frac{1}{2} \Omega[\cos \chi \cos (\varphi-\eta)-i \sin (\varphi-\eta)]$. Although $\mathbf{A}+\mathbf{K}$ is a diagonal matrix, $\mathbf{A}$ and $\mathbf{K}$ are both nondiagonal in our scheme. Specifically, A does represent a non-Abelian connection with nonvanishing commutation relation $\left[\mathbf{A}(t), \mathbf{A}\left(t^{\prime}\right)\right] \neq 0$, which proves the non-Abelian nature of the gate in Eq. (4) [27]. In addition, we emphasize that the auxiliary states $\left|\phi_{1,2}(t)\right\rangle$ in our model are chosen generally for the resonate two-level system and thus the choice of auxiliary states does not change the above results.

Now, to construct the NNGQC gate, one simple parameter set of choice is found to be $\chi(t)=\Omega_{0} t-\chi_{0}, \quad \eta(t)=$ $\phi_{1} \epsilon(t)+\phi_{0}$, where the step function $\epsilon(t)$ satisfies $\epsilon=0$ with $t \in\left[0, \frac{\chi_{0}}{\Omega_{0}}\right]$ and $\epsilon=1$ with $t \in\left[\frac{\chi_{0}}{\Omega_{0}}, \tau\right]$ and $\Omega_{0}, \phi_{1}, \phi_{0}$ and $\chi_{0}$ are constants. With the settings, we can obtain the initial and final value $\eta_{ \pm}$and $\chi_{ \pm}$as $\eta_{+}=\phi_{1}+2 \phi_{0}, \eta_{-}=\phi_{1}, \chi_{+}=$ $\Omega_{0} \tau-2 \chi_{0}, \chi_{-}=\Omega_{0} \tau$. Meanwhile, the geometirc phase is taken by $\gamma=\int_{0}^{\tau} \frac{1}{2} \dot{\eta} \cos \chi d t=\frac{\phi_{1}}{2}$. For $U_{1}$ gate, the control parameters are chosen as $\chi_{0}=\pi / 2, \phi_{0}=-\pi / 2, \phi_{1}=\pi / 2$, and $\tau=\frac{\pi}{\Omega_{0}}$.In sharp contrast to the conventional NGQC schemes to construct $U_{1}$ [42-46] that requires evolution time being $2 \tau$, by choosing the same maximum Rabi frequency, we found that the required gate time of $\mathrm{NNGQC}$ to construct $U_{1}$ is $\tau$ [as shown in Fig. 2(a)], which is reduced by $50 \%$ (see Appendix A for details).

\section{GATE PERFORMANCE AND ROBUSTNESS}

The performance of the $U_{1}$ gate can be simulated by using the Lindblad master equation [86]. In our simulation, we have used the following set of experimental parameters [8-10]. The Rabi frequency, decay and dephasing rates are set
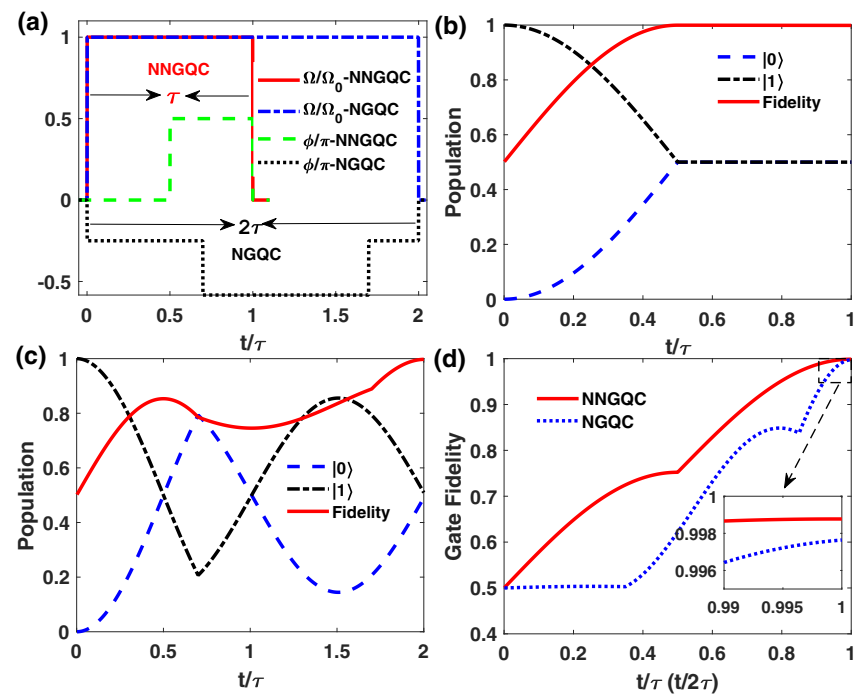

FIG. 2. (a) The Rabi frequency $\Omega(t)$ and phase $\varphi(t)$ of $U_{1}$ gate for NNGQC and NGQC. State population and state fidelity of (b) NNGQC and (c) NGQC with the initial state being $|0\rangle$. (d) Gate fidelity of $U_{1}$ as a function of $t / \tau(t / 2 \tau)$.

as $\Omega_{0}=2 \pi \times 6.25 \mathrm{kHz}, \gamma_{1} \approx 2 \Omega_{0} \times 10^{-4}$, and $\gamma_{2} \approx 2 \Omega_{0} \times$ $10^{-3}$, corresponding to $T_{1}=590 \mathrm{~ms}$ and $T_{2}=50 \mathrm{~ms}$. Suppose that the qubit is initially prepared in the $|\psi(0)\rangle=|0\rangle$ state, the time-dependence of the state populations and the state fidelity $F=\left|\left\langle\psi_{I} \mid \psi(\tau)\right\rangle\right|^{2}$ of realizing the $U_{1}$ gate for NNGQC and NGQC are depicted in Figs. 2(b) and 2(c), where the state fidelities of NNGQC and NGQC are obtained to be $99.87 \%$ and $99.75 \%$, respectively. Furthermore, we have also investigated the gate fidelity of $U_{1}$ defined by $F=(1 / 2 \pi) \int_{0}^{2 \pi}\left\langle\psi_{I}|\rho| \psi_{I}\right\rangle d \Theta$ for initial states of the form $|\psi\rangle=\cos \Theta|0\rangle+\sin \Theta|1\rangle$, where a total of 1001 different values of $\Theta$ were uniformly chosen in the range of $[0,2 \pi]$, as shown in Fig. 2(d). We found that the gate error $(1-F)$ of NNGQC can be reduced by as much as $50 \%$ compared with the gate error of NGQC $(0.24 \%)$.

Now, we start to demonstrate the robustness of our scheme. We first consider the robustness of our NNGQC against Rabi errors and assume the amplitudes of control pulse to vary in the range of $\Omega_{0} \rightarrow(1+\zeta) \Omega_{0}$ with the error fraction $\zeta \in$ $[-0.1,0.1]$. Next, we take the detuning noise to be $\Delta \sigma_{z}$ with $\Delta=\delta \Omega_{0}$ being static and the fraction is $\delta \in[-0.1,0.1]$. Comparing our NNGQC with the conventional NGQC methods, we plot the performance of the same geometric gate with the same pulse error. As shown in Figs. 3(a), 3(b), and 3(c), the NNGQC is always more robust than the NGQC gate. Furthermore, we also simulated the gate fidelity as a function of decay rate and dephasing rate $\gamma_{1}$ and $\gamma_{2}$. For above two schemes as shown in Figs. 3(d), 3(e), and 3(f), our scheme of NNGQC can greatly suppress the decoherence effect comparing with the conventional NGQC.

To further show the noise-resilient geometric feature of our NNGQC, we also take the above noises to compare the performance of our geometric gate with that of the corresponding dynamical gate (DG) $[87,88]$ as shown in Appendix B. From the numerical result, we can clearly see that our NNGQC 


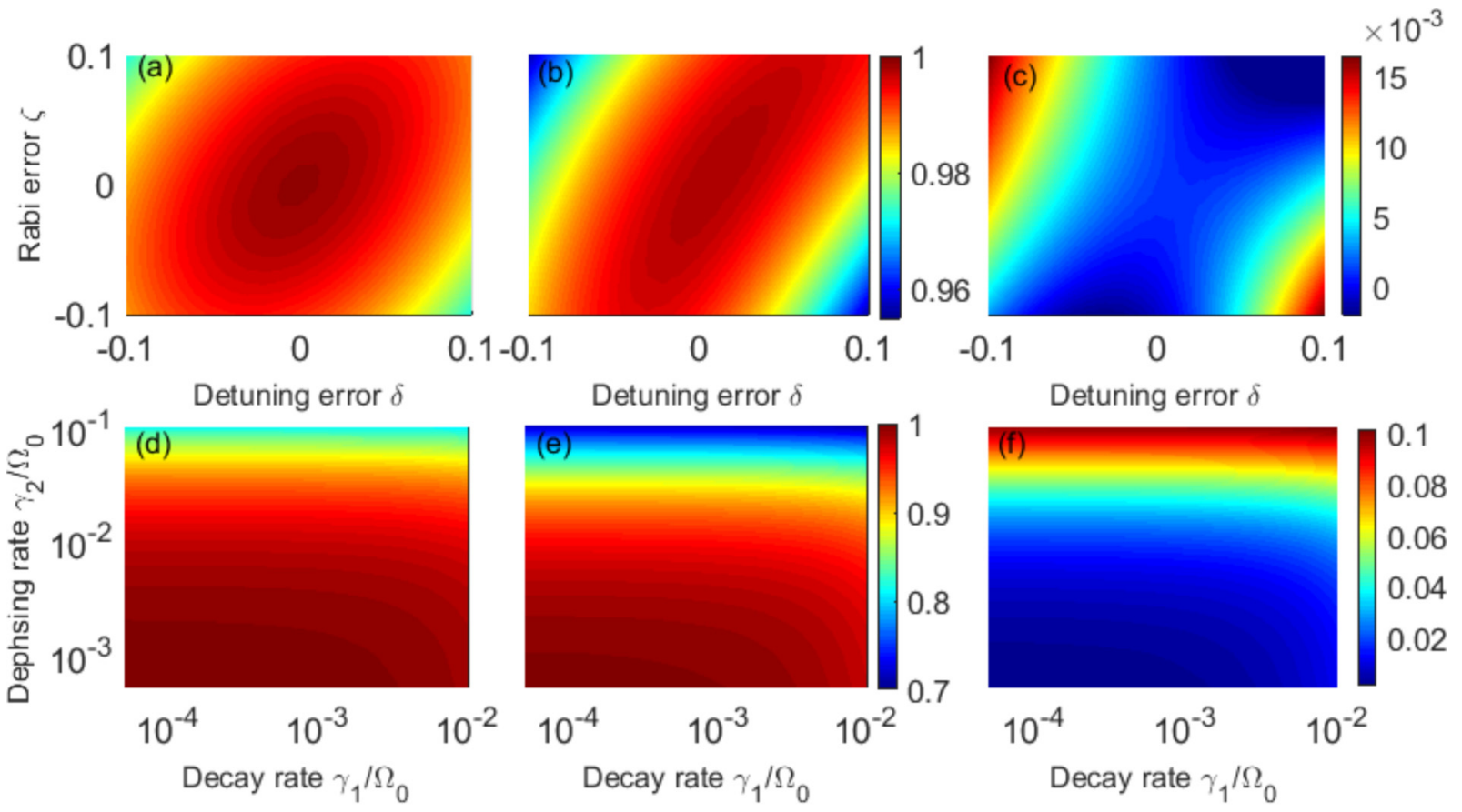

FIG. 3. The performance of $U_{1}$ gate under imperfections. Gate fidelities of (a) NNGQC, (b) NGQC under the Rabi error $\zeta$ and detuning error $\delta$. Panel (c) is the difference between (a) and (b). The gate fidelities for (d) NNGQC, (e) NGQC, and (f) difference as a function of decay rate $\gamma_{1}$ and dephasing rate $\gamma_{2}$, respectively.

scheme is more robust against both the pulse control error and decoherence error than DG scheme.

\section{NONTRIVIAL TWO-QUBIT GATE}

In this section, we proceed to implement nontrivial twoqubit Rydberg quantum gates free from blockade error with the pulse similar to that designed in single-qubit case. As shown in Fig. 4, we consider two ${ }^{133} \mathrm{Cs}$ atoms with magnetically insensitive "clock" states encoding $|0\rangle \equiv \mid 6 S_{1 / 2}, F=$ $\left.3, m_{F}=0\right\rangle$ and $|1\rangle \equiv\left|6 S_{1 / 2}, F=4, m_{F}=0\right\rangle$ [83]. The Rydberg state is chosen as $|R\rangle \equiv\left|61 S_{1 / 2}\right\rangle$. And the $C_{6}$ parameter can be evaluated as $126 \mathrm{GHz} \mu \mathrm{m}^{6}$ [89].

The basic process of two-qubit gate is shown in Fig. 4, where the control in resonant interaction but the target atom is large-detuning interaction. The required three steps are as follows.

Step $(i)$. Turn on the laser on control atom with Hamiltonian

$$
H_{c}=\frac{\Omega_{1}(t)}{2}|1\rangle\langle R|+\text { H.c. },
$$

where $\quad \Omega_{1}(t) \equiv\left|\Omega_{1}(t)\right| e^{i \varphi_{1}(t)}$. We $\quad$ set $\quad \varphi_{1}(t)=0 \quad$ and $\int \Omega_{1}(t) d t=\pi$ in step (i).

Step (ii). Turn off the laser on control atom and turn on lasers with Rabi frequencies $\Omega_{S}$ and $\Omega_{P}$ on target atom. The Hamiltonian is

$$
H_{t}=\Delta|R\rangle\left\langleR \left|+\frac{1}{2}\left[\Omega_{S}(t)|0\rangle+\Omega_{P}(t)|1\rangle\right]\langle R|+\text { H.c. },\right.\right.
$$

where $\Omega_{S}(t) \equiv\left|\Omega_{S}(t)\right| e^{i \varphi_{S}(t)}$ and $\Omega_{P}(t) \equiv\left|\Omega_{P}(t)\right| e^{i \varphi_{P}(t)}$. Besides, the RRI Hamiltonian

$$
H_{V}=V|R R\rangle\langle R R|
$$

may be in existence conditioned on the control atom is excited or not. We use $|m n\rangle\langle m n|$ to denote the abbreviation of $|m\rangle\langle m|\otimes| n\rangle\langle n|$ here and throughout the manuscript for simplify. Thus, the dynamical process can be classified as two cases in Figs. 4(b) and 4(c), respectively, depend whether the control atom is not excited or excited.
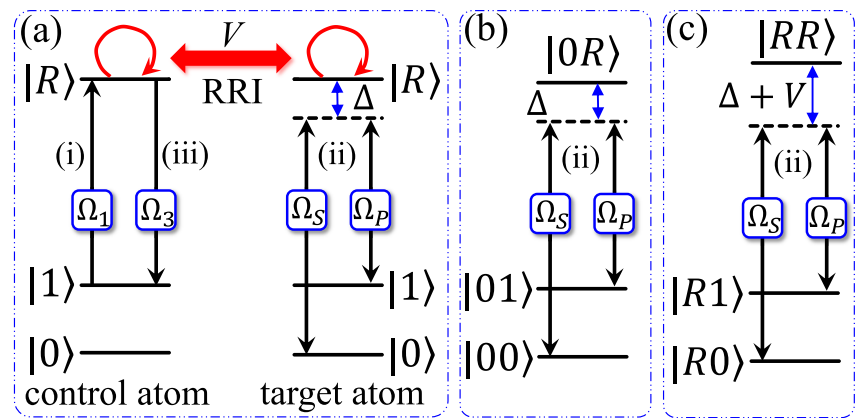

FIG. 4. (a) Illustration of the two-qubit NNGQC gate based on unconventional Rydberg blockade with single-atom basis. $V$ denotes the RRI strength. For control atom, $|1\rangle$ is coupled with $|R\rangle$ through two-photon process with Rabi frequency $\Omega_{1}\left(\Omega_{3}\right)$ in step (i) [(iii)]. For target atom, $|0\rangle(|1\rangle)$ is coupled with $|R\rangle$ with Rabi frequency $\Omega_{S}\left(\Omega_{P}\right)$ and detuning $\Delta$ via two-photon process in step (ii). $\Omega_{j}=$ $\left|\Omega_{j}\right| e^{i \varphi_{j}}$ with $j=1,3, S, P$. (b) [(c)] Dynamical process of step (ii) under two-atom basis in the without (with) RRI. For conventional blockade, the dynamical process in panel (b) is resonant while in panel (c) is detuned by $V$ and always be discarded. However, in our scheme both of the dynamical processes in panels (b) and (c) are used for the gate, and thus one can call it "unconventional Rydberg blockade." 
The effective Hamiltonian in Fig. 4(b) can be calculated as

$$
H_{\mathrm{eff}, 1}=\frac{\Omega_{\mathrm{eff}, 1}}{2}|00\rangle\langle 01|+\text { H.c. },
$$

where $\Omega_{\mathrm{eff}, 1}=\Omega_{S} \Omega_{P}^{*} /(2 \Delta)$ and the stark shifts are vanished when $\left|\Omega_{S}\right|=\left|\Omega_{P}\right|$. Similarly, the effective Hamiltonian in Fig. 4(c) would be

$$
H_{\mathrm{eff}, 2}=\frac{\Omega_{\mathrm{eff}, 2}}{2}|R 0\rangle\langle R 1|+\text { H.c. },
$$

where $\Omega_{\text {eff }, 2}=\Omega_{S} \Omega_{P}^{*} /[2(\Delta+V)]$ and the stark shifts are also vanished when $\left|\Omega_{S}\right|=\left|\Omega_{P}\right|$.

Equations (9) and (10) have similar form as Eq. (1). Thus, we can use the similar pulses to construct the noncyclic nonadiabatic geometric operations. That is, in step (ii), one can get the operation

$$
\mathcal{U}_{2}=|0\rangle_{c}\left\langle 0\left|\otimes U\left(\theta_{1}, \alpha_{1}, \beta_{1}\right)+\right| R\right\rangle_{c}\langle R| \otimes U\left(\theta_{2}, \alpha_{2}, \beta_{2}\right),
$$

with suitable laser parameters.

Step(iii). Turn off the lasers on target atom and at the same time turn on laser with Rabi frequency $\Omega_{3}(t)$ on control atom. If $\left|\Omega_{3}\left(t-t_{2}-t_{1}\right)\right|=\left|\Omega_{1}(t)\right|$ and $\varphi_{3}=\pi$, in which $t_{1(2)}$ denotes the evolution time in step (i) [(ii)], one can get the the whole evolution operator as

$\mathcal{U}=|0\rangle_{c}\left\langle 0\left|\otimes U\left(\theta_{1}, \alpha_{1}, \beta_{1}\right)+\right| 1\right\rangle_{c}\langle 1| \otimes U\left(\theta_{2}, \alpha_{2}, \beta_{2}\right)$.

Therefore, in general, we know that Eq. (12) represents a nontrivial two-qubit entangled gate, since $U$ in subspace $\{|00\rangle,|01\rangle\}$ and $\{|10\rangle,|11\rangle\}$ is different.

When $\theta_{1}=\pi / 2, \alpha_{1}=\pi / 2, \beta_{1}=\pi / 2$ and $\Delta=V$, we obtain two-qubit entangled gate with matrix representation as

$$
\mathcal{U}=\left(\begin{array}{cccc}
0 & i & 0 & 0 \\
-i & 0 & 0 & 0 \\
0 & 0 & \frac{e^{i \pi / 4}}{\sqrt{2}} & \frac{e^{i \pi / 4}}{\sqrt{2}} \\
0 & 0 & \frac{e^{i \pi / 4}}{\sqrt{2}} & \frac{-e^{i \pi / 4}}{\sqrt{2}}
\end{array}\right) .
$$

Therefore, our scheme is sufficient for universal quantum computation when assisted by a combination of the single-and two-qubit gate.

To evaluate the performance of two-qubit entangled gate, we take the parameters from the state-of-art experiments as the Rabi frequency $\Omega_{0}=\Omega_{1}=2 \pi \times 10 \mathrm{MHz}$ and the detuning $\Delta \approx 17 \Omega_{0}$, where $\Omega_{P}$ and $\Omega_{S}$ are governed by the Eq. (2) in maintext. As shown in Fig. 5(a), we plot the state populations and the state fidelity of the two-qubit gate with the initial state $|\psi(0)\rangle=\frac{1}{2}(|00\rangle+|01\rangle+\sqrt{2}|10\rangle)$, where the state fidelity is obtained to be $99.97 \%$ without considering relaxation. Moreover, we have also investigated the gate infidelity $[90,91]$ of two-qubit $1-F$ as a function of decay rate and dephasing rate of Rydberg state as shown in Fig. 5(b), and found that our two-qubit geometric gate is robust against decoherence from the environmental noises.

\section{CONCLUSION}

In summary, we have presented a new framework of NNGQC, which universal nonadiabatic geometric gates can be constructed via noncyclic non-Abelian geometric phase. Comparing with conventional NGQC, NNGQC can further
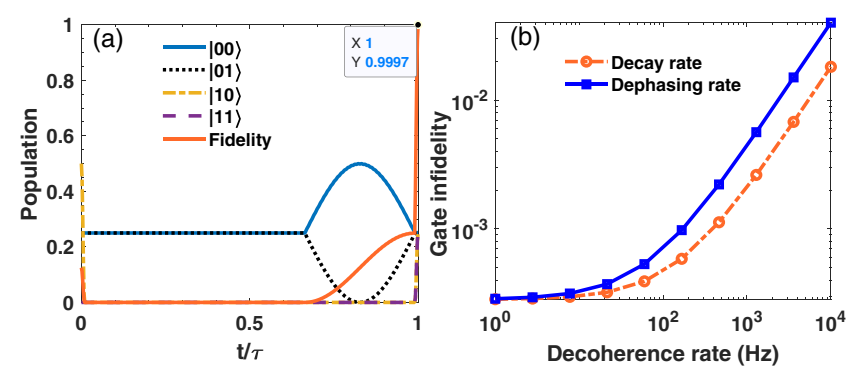

FIG. 5. (a) State population and state fidelity of two-qubit gate with the initial state being $\frac{1}{2}(|00\rangle+|01\rangle+\sqrt{2}|10\rangle)$. (b) Gate infidelity as a function of decay rate and dephasing rate for Rydberg state.

reduce the geometric gate time beyond the limitation of cyclic condition. Consequently, our proposal is more robust against the decay and dephasing effects from the environmental decoherence. Moreover, we construct a nontrivial two-qubit geometric gate via RRI-induced large detuning process seriously without discarding the process induced by RRI-induced "blockade" terms. Therefore, our scheme provides a promising way towards fault-tolerant quantum computation for neutral-atom-based quantum system.

\section{ACKNOWLEDGMENTS}

This work is supported by the Key-Area Research and Development Program of Guangdong Province (Grant No. 2018B030326001), the National Natural Science Foundation of China (Grants No. 11875160, No. 11874156, and No. 11804308), the Natural Science Foundation of Guangdong Province (Grant No. 2017B030308003), the National Key R\& D Program of China (Grant No. 2016YFA0301803), the Guangdong Innovative and Entrepreneurial Research Team Program (Grant No. 2016ZT06D348), the Economy, Trade and Information Commission of Shenzhen Municipality (Grant No. 201901161512), the Science, Technology and Innovation Commission of Shenzhen Municipality (Grants No. JCYJ20170412152620376，No. JCYJ20170817105046702, and No. KYTDPT20181011104202253).

\section{APPENDIX A: NONADIABATIC GEOMETIRC QUANTUM COMPUTATION}

Here, we shall succinctly derive NGQC [42-46] in our framework to provide a unified view on geometric quantu computation. For conventional NGQC, it should satisfy the cyclic condition as $\left|\phi_{1}(\tau)\right\rangle=\left|\phi_{1}(0)\right\rangle$. Consequently, the Rabi frequency is governed by the following equation

$$
\int_{0}^{T} \Omega(t) d t=2 \pi,
$$

where $T$ denotes the gate time. In this way, the cyclic nonadiabatic geometric gate becomes

$$
\begin{aligned}
U(\tau, 0) & =e^{i \gamma}\left|\phi_{1}(0)\right\rangle\left\langle\phi_{1}(0)\left|+e^{-i \gamma}\right| \phi_{2}(0)\right\rangle\left\langle\phi_{2}(0)\right| \\
& =\cos \gamma+i \sin \gamma\left(\begin{array}{cc}
\cos \mu & \sin \mu e^{-i \eta_{0}} \\
\sin \mu e^{i \eta_{0}} & -\cos \mu
\end{array}\right) \\
& =e^{i \gamma \vec{n} \cdot \vec{\sigma}},
\end{aligned}
$$



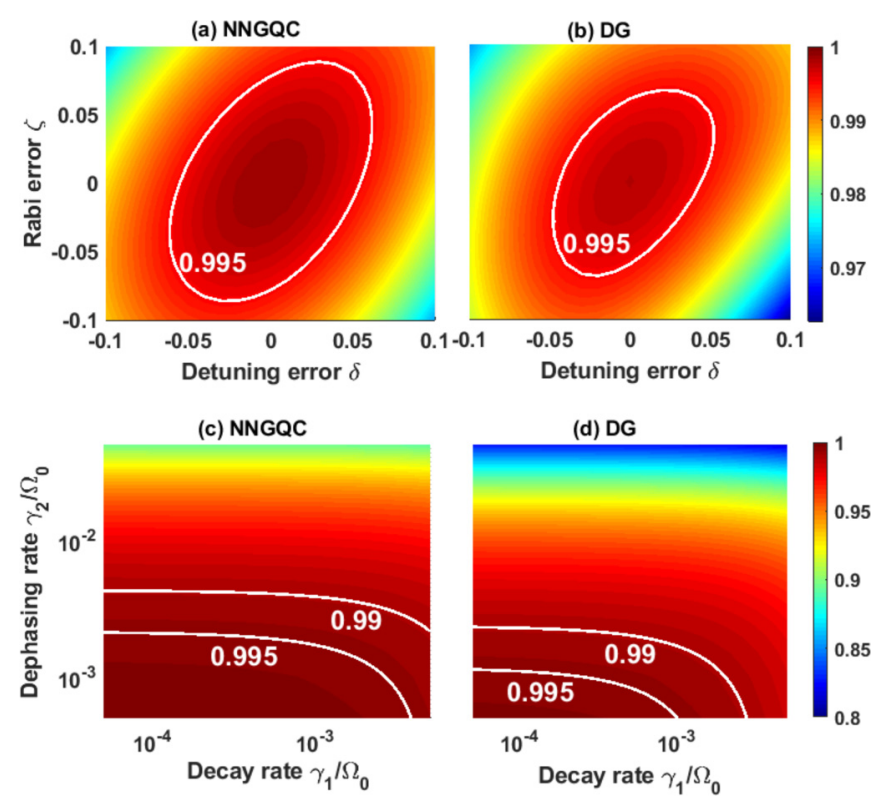

FIG. 6. Gate fidelities of (a) NNGQC and (b) DG under the Rabi error $\zeta$ and detuning error $\delta$. The gate fidelities for (c) NNGQC and (d) DG as a function of decay rate $\gamma_{1}$ and dephasing rate $\gamma_{2}$, respectively.

where $\mu=\chi(0)$ and $\eta_{0}=\eta(0)$ denotes the initial value of $\chi$ and $\eta$ at time $t=0 ; \vec{n}=\left(\sin \mu \cos \eta_{0}, \sin \mu \sin \eta_{0}, \cos \mu\right)$, and $\vec{\sigma}$ are the Pauli matrices.

To realize the $U_{1}$ gate of NGQC, we take parameters as $\gamma=$ $3 \pi / 4, \eta_{0}=\pi / 4$, and $\mu=7 \pi / 20$ corresponding the Rabi frequency $\Omega(t)=\Omega_{0}$ and the evolution time $T=2 \pi / \Omega_{0}=2 \tau$, as shown in Fig. 2(a).

\section{APPENDIX B: ROBUSTNESS OF NNGQC VERSUS DYNAMICAL GATE}

Before comparing the robustness of our NNGQC gate with the dynamical gate (DG) $[87,88]$, let's briefly talk about how to realize the $U_{1}$ dynamical gate. When this Hamiltonian $H$ is time-independent, we can obtain the evolution operator as

$$
U_{d g}\left(\Omega \tau_{1}, \varphi\right)=\left(\begin{array}{cc}
\cos \left(\frac{\Omega \tau_{1}}{2}\right) & -i e^{-i \varphi} \sin \left(\frac{\Omega \tau_{1}}{2}\right) \\
-i e^{i \varphi} \sin \left(\frac{\Omega \tau_{1}}{2}\right) & \cos \left(\frac{\Omega \tau_{1}}{2}\right)
\end{array}\right)
$$

In general, any single-qubit gate can be realized by the evolution operator. We note that Eq. (B1) is X-rotation $U_{d g}=$ $X_{\Omega \tau_{1}}$ when $\varphi=0$. To realize a Z-rotation gate $U_{d g}=Z_{\phi}$, we need to take two sequential evolutions $U_{d g}(\pi, \phi / 2)$ and $U_{d g}(\pi,-\phi / 2)$. In this way, the general dyamical gate is given by

$$
U_{d g}=Z_{\phi} X_{\Omega \tau_{1}} Z_{\alpha}
$$

For the $U_{1}$ dynamical gate, we take parameters as $\Omega(t)=\Omega_{0}$, $\tau_{1}=\pi / 2 \Omega_{0}=\frac{1}{2} \tau, \phi=0, \alpha=-\pi / 2$, and $T=5 \tau / 2$.

Comparison of the robustness against the detuning error and Rabi frequency, of all the two implementations are shown in Figs. 6(a) and 6(b). From the numerical result, we can clearly see that the NNGQC scheme is more robust against the pulse control errors than the corresponding DG scheme. Furthermore, we also consider comparison of the robustness against the decay rate and dephasing rate caused by environmental noise. From the Figs. 6(c) and 6(d), we know that our scheme of NNGQC can suppress the decoherence effect comparing with the DG.
[1] G. K. Brennen, C. M. Caves, P. S. Jessen, and I. H. Deutsch, Quantum Logic Gates in Optical Lattices, Phys. Rev. Lett. 82, 1060 (1999).

[2] D. S. Weiss and M. Saffman, Quantum computing with neutral atoms, Phys. Today 70(7), 44 (2017).

[3] T. F. Gallagher, Rydberg Atoms (Cambridge University Press, Cambridge, UK, 1994).

[4] D. Jaksch, J. I. Cirac, P. Zoller, S. L. Rolston, R. Côté, and M. D. Lukin, Fast Quantum Gates for Neutral Atoms, Phys. Rev. Lett. 85, 2208 (2000).

[5] M. D. Lukin, M. Fleischhauer, R. Côté, L. M. Duan, D. Jaksch, J. I. Cirac, and P. Zoller, Dipole Blockade and Quantum Information Processing in Mesoscopic Atomic Ensembles, Phys. Rev. Lett. 87, 037901 (2001).

[6] M. Saffman, T. G. Walker, and K. Mølmer, Quantum information with Rydberg atoms, Rev. Mod. Phys. 82, 2313 (2010).

[7] D. Schrader, I. Dotsenko, M. Khudaverdyan, Y. Miroshnychenko, A. Rauschenbeutel, and D. Meschede, Neutral Atom Quantum Register, Phys. Rev. Lett. 93, 150501 (2004).

[8] S. Olmschenk, R. Chicireanu, K. D. Nelson, and J. V. Porto, Randomized benchmarking of atomic qubits in an optical lattice, New J. Phys. 12, 113007 (2010).
[9] T. Xia, M. Lichtman, K. Maller, A. W. Carr, M. J. Piotrowicz, L. Isenhower, and M. Saffman, Randomized Benchmarking of Single-Qubit Gates in a 2D Array of Neutral-Atom Qubits, Phys. Rev. Lett. 114, 100503 (2015).

[10] Y. Wang, X. Zhang, T. A. Corcovilos, A. Kumar, and D. S. Weiss, Coherent Addressing of Individual Neutral Atoms in a 3D Optical Lattice, Phys. Rev. Lett. 115, 043003 (2015); Y. Wang, A. Kumar, T.-Y. Wu, and D. S. Weiss, Single-qubit gates based on targeted phase shifts in a 3D neutral atom array, Science 352, 1562 (2016).

[11] J. H. Lee, E. Montano, I. H. Deutsch, and P. S. Jessen, Robust site-resolvable quantum gates in an optical lattice via inhomogeneous control, Nat. Commum. 4, 2027 (2013).

[12] A. Smith, B. E. Anderson, H. Sosa-Martinez, C. A. Riofrío, Ivan H. Deutsch, and Poul S. Jessen, Quantum Control in the Cs $6 S_{1 / 2}$ Ground Manifold using Radio-Frequency and Microwave Magnetic Fields, Phys. Rev. Lett. 111, 170502 (2013).

[13] L. Isenhower, E. Urban, X. L. Zhang, A. T. Gill, T. Henage, T. A. Johnson, T. G. Walker, and M. Saffman, Demonstration of a Neutral Atom Controlled-NOT Quantum Gate, Phys. Rev. Lett. 104, 010503 (2010).

[14] T. Wilk, A. Gaëtan, C. Evellin, J. Wolters, Y. Miroshnychenko, P. Grangier, and A. Browaeys, Entanglement of Two Individual 
Neutral Atoms Using Rydberg Blockade, Phys. Rev. Lett. 104, 010502 (2010).

[15] K. M. Maller, M. T. Lichtman, T. Xia, Y. Sun, M. J. Piotrowicz, A. W. Carr, L. Isenhower, and M. Saffman, Rydberg-blockade controlled-not gate and entanglement in a two-dimensional array of neutral-atom qubits, Phys. Rev. A 92, 022336 (2015).

[16] Y. Zeng, P. Xu, X. He, Y. Liu, M. Liu, J. Wang, D. J. Papoular, G. V. Shlyapnikov, and M. Zhan, Entangling Two Individual Atoms of Different Isotopes via Rydberg Blockade, Phys. Rev. Lett. 119, 160502 (2017).

[17] C. J. Picken, R. Legaie, K. McDonnell, and J. D. Pritchard, Entanglement of neutral-atom qubits with long ground-Rydberg coherence times, Quan. Sci. Tech. 4, 015011 (2018).

[18] H. Levine, A. Keesling, A. Omran, H. Bernien, S. Schwartz, A. S. Zibrov, M. Endres, M. Greiner, V. Vuletić, and M. D. Lukin, High-Fidelity Control and Entanglement of RydbergAtom Qubits, Phys. Rev. Lett. 121, 123603 (2018).

[19] H. Levine, A. Keesling, G. Semeghini, A. Omran, T. T. Wang, S. Ebadi, H. Bernien, M. Greiner, V. Vuletić, H. Pichler, and M. D. Lukin, Parallel Implementation of High-Fidelity Multiqubit Gates with Neutral Atoms, Phys. Rev. Lett. 123, 170503 (2019).

[20] T. M. Graham, M. Kwon, B. Grinkemeyer, Z. Marra, X. Jiang, M. T. Lichtman, Y. Sun, M. Ebert, and M. Saffman, Rydberg Mediated Entanglement in a Two-Dimensional Neutral Atom Qubit Array, Phys. Rev. Lett. 123, 230501 (2019).

[21] I. S. Madjarov, J. P. Covey, A. L. Shaw et al., High-fidelity entanglement and detection of alkaline-earth Rydberg atoms, Nat. Phys. 16, 857 (2020).

[22] E. Sjöqvist, Trend: A new phase in quantum computation, Physics 1, 35 (2008); Geometric phases in quantum information, Int. J. Quantum Chem. 115, 1311 (2015).

[23] P. Zanardi and M. Rasetti, Holonomic quantum computation, Phys. Lett. A 264, 94 (1999); J. Pachos, P. Zanardi, and M. Rasetti, Non-Abelian Berry connections for quantum computation, Phys. Rev. A 61, 010305(R) (1999).

[24] M. V. Berry, Quantal phase factors accompanying adiabatic changes, Proc. R. Soc. Lond. A 392, 45 (1984).

[25] F. Wilczek and A. Zee, Appearance of Gauge Structure in Simple Dynamical Systems, Phys. Rev. Lett. 52, 2111 (1984).

[26] Y. Aharonov and J. Anandan, Phase Change During a Cyclic Quantum Evolution, Phys. Rev. Lett. 58, 1593 (1987).

[27] J. Anandan, Non-adiabatic non-Abelian geometric phase, Phys. Lett. A 133, 171 (1988).

[28] S.-L. Zhu and P. Zanardi, Geometric quantum gates that are robust against stochastic control errors, Phys. Rev. A 72, 020301(R) (2005).

[29] S. Berger, M. Pechal, A. A. Abdumalikov, Jr., C. Eichler, L. Steffen, A. Fedorov, A. Wallraff, and S. Filipp, Exploring the effect of noise on the Berry phase, Phys. Rev. A 87, 060303(R) (2013).

[30] G. D. Chiara and G. M. Palma, Berry Phase for a Spin-1/2 Particle in a Classical Fluctuating Field, Phys. Rev. Lett. 91, 090404 (2003).

[31] P. J. Leek, J. M. Fink, A. Blais, R. Bianchetti, M. Göppl, J. M. Gambetta, D. I. Schuster, L. Frunzio, R. J. Schoelkopf, and A. Wallraff, Observation of Berry's phase in a solid state qubit, Science 318, 1889 (2007).
[32] S. Filipp, J. Klepp, Y. Hasegawa, C. Plonka-Spehr, U. Schmidt, P. Geltenbort, and H. Rauch, Experimental Demonstration of the Stability of Berry's Phase for a Spin-1/2 Particle, Phys. Rev. Lett. 102, 030404 (2009).

[33] J. T. Thomas, M. Lababidi, and M. Tian, Robustness of singlequbit geometric gate against systematic error, Phys. Rev. A 84, 042335 (2011).

[34] M. Johansson, E. Sjöqvist, L. M. Andersson, M. Ericsson, B. Hessmo, K. Singh, and D. M. Tong, Robustness of non-adiabatic holonomic gates, Phys. Rev. A 86, 062322 (2012).

[35] L. M. Duan, J. I. Cirac, and P. Zoller, Geometric manipulation of trapped ions for quantum computation, Science 292, 1695 (2001).

[36] L.-A. Wu, P. Zanardi, and D. A. Lidar, Holonomic Quantum Computation in Decoherence-Free Subspaces, Phys. Rev. Lett. 95, 130501 (2005).

[37] J. A. Jones, V. Vedral, A. Ekert, and G. Castagnoli, Geometric quantum computation using nuclear magnetic resonance, Nature 403, 869 (2000).

[38] Y.-Y. Huang, Y.-K. Wu, F. Wang, P.-Y. Hou, W.-B. Wang, W.-G. Zhang, W.-Q. Lian, Y.-Q. Liu, H.-Y. Wang, H.-Y. Zhang et al., Experimental Realization of Robust Geometric Quantum Gates with Solid-State Spins, Phys. Rev. Lett. 122, 010503 (2019).

[39] Wang Xiang-Bin and M. Keiji, Nonadiabatic Conditional Geometric Phase Shift with NMR, Phys. Rev. Lett. 87, 097901 (2001).

[40] S.-L. Zhu and Z. D. Wang, Implementation of Universal Quantum Gates Based on Nonadiabatic Geometric Phases, Phys. Rev. Lett. 89, 097902 (2002).

[41] Z.-T. Liang, X. Yue, Q. Lv, Y.-X. Du, W. Huang, H. Yan, and S.-L. Zhu, Proposal for implementing universal superadiabatic geometric quantum gates in nitrogen-vacancy centers, Phys. Rev. A 93, 040305(R) (2016).

[42] P. Z. Zhao, X.-D. Cui, G. F. Xu, E. Sjöqvist, and D. M. Tong, Rydberg-atom-based scheme of nonadiabatic geometric quantum computation, Phys. Rev. A 96, 052316 (2017).

[43] T. Chen and Z.-Y. Xue, Nonadiabatic Geometric Quantum Computation with Parametrically Tunable Coupling, Phys. Rev. Appl. 10, 054051 (2018).

[44] C. Zhang, T. Chen, S. Li, and Z.-Y. Xue, High-fidelity geometric gate for silicon-based spin qubits, Phys. Rev. A 101, 052302 (2020).

[45] Y. Xu et al., Experimental Implementation of Universal Nonadiabatic Geometric Quantum Gates in a Superconducting Circuit, Phys. Rev. Lett. 124, 230503 (2020).

[46] P. Z. Zhao, Z. Dong, Z. Zhang, G. Guo, D. M. Tong, and Y. Yin, Experimental realization of nonadiabatic geometric gates with a superconducting Xmon qubit, arXiv:1909.09970.

[47] E. Sjöqvist, D. M. Tong, L. M. Andersson, B. Hessmo, M. Johansson, and K. Singh, Non-adiabatic holonomic quantum computation, New J. Phys. 14, 103035 (2012).

[48] G. F. Xu, J. Zhang, D. M. Tong, E. Sjöqvist, and L. C. Kwek, Nonadiabatic Holonomic Quantum Computation in Decoherence-Free Subspaces, Phys. Rev. Lett. 109, 170501 (2012).

[49] B.-J. Liu, X.-K. Song, Z.-Y. Xue, X. Wang, and M.-H. Yung, Plug-and-Play Approach to Nonadiabatic Geometric Quantum Gates, Phys. Rev. Lett. 123, 100501 (2019). 
[50] B.-J. Liu, Z.-Y. Xue, and M.-H. Yung, Brachistochronic nonadiabatic holonomic quantum control, arXiv:2001.05182.

[51] Z.-Y. Xue, F.-L. Gu, Z.-P. Hong, Z.-H. Yang, D.-W. Zhang, Y. Hu, and J. Q. You, Nonadiabatic Holonomic Quantum Computation with Dressed-State Qubits, Phys. Rev. Appl. 7, 054022 (2017).

[52] J. Zhou, B. J. Liu, Z. P. Hong, and Z. Y. Xue, Fast holonomic quantum computation based on solid-state spins with all-optical control, Sci. China-Phys. Mech. Astron. 61, 010312 (2018),

[53] V. Azimi Mousolou, Electric nonadiabatic geometric entangling gates on spin qubits, Phys. Rev. A 96, 012307 (2017).

[54] X.-K. Song, H. Zhang, Q. Ai, J. Qiu, and F.-G. Deng, Shortcuts to adiabatic holonomic quantum computation in decoherencefree subspace with transitionless quantum driving algorithm, New J. Phys. 18, 023001 (2016).

[55] B.-J. Liu, Z.-H. Huang, Z.-Y. Xue, and X.-D. Zhang, Superadiabatic holonomic quantum computation in cavity QED, Phys. Rev. A 95, 062308 (2017).

[56] Y.-H. Kang, Z.-C. Shi, B.-H. Huang, J. Song, and Y. Xia, Flexible scheme for the implementation of nonadiabatic geometric quantum computation, Phys. Rev. A 101, 032322 (2020).

[57] A. A. Abdumalikov, J. M. Fink, K. Juliusson, M. Pechal, S. Berger, A. Wallraff, and S. Filipp, Experimental realization of non-Abelian non-adiabatic geometric gates, Nature 496, 482 (2013).

[58] C. Song, S.-B. Zheng, P. Zhang, K. Xu, L. Zhang, Q. Guo, W. Liu, D. Xu, H. Deng, K. Huang et al., Continuous-variable geometric phase and its manipulation for quantum computation in a superconducting circuit, Nat. Commun. 8, 1061 (2017).

[59] T. Yan et al., Experimental Realization of Non-Adiabatic Shortcut to Non-Abelian Geometric Gates, Phys. Rev. Lett. 122, 080501 (2019).

[60] Y. Xu et al., Single-Loop Realization of Arbitrary Nonadiabatic Holonomic Single-Qubit Quantum Gates in a Superconducting Circuit, Phys. Rev. Lett. 121, 110501 (2018).

[61] Z. Han et al., Experimental realization of universal time-optimal non-Abelian geometric gates, arXiv:2004.10364.

[62] G. Feng, G. Xu, and G. Long, Experimental Realization of Nonadiabatic Holonomic Quantum Computation, Phys. Rev. Lett. 110, 190501 (2013).

[63] H. Li, L. Yang, and G. Long, Experimental realization of singleshot nonadiabatic holonomic gates in nuclear spins, Sci. China: Phys., Mech. Astron. 60, 080311 (2017).

[64] Z. Zhu, T. Chen, X. Yang, J. Bian, Z.-Y. Xue, and X. Peng, Single-Loop and Composite-Loop Realization of Nonadiabatic Holonomic Quantum Gates in a Decoherence-Free Subspace, Phys. Rev. Appl. 12, 024024 (2019).

[65] Y. Li, T. Xin, C. Qiu, K. Li, G. Liu, J. Li, Y. Wan, and D. Lu, Dynamical-invariant-based holonomic quantum gates: Theory and experiment, arXiv:2003.09848.

[66] C. $\mathrm{Zu}$ et al., Experimental realization of universal geometric quantum gates with solid-state spins, Nature (London) 514, 72 (2014).

[67] S. Arroyo-Camejo, A. Lazariev, S. W. Hell, and G. Balasubramanian, Room temperature high-fidelity holonomic single-qubit gate on a solid-state spin, Nat. Commun. 5, 4870 (2014).

[68] Y. Sekiguchi, N. Niikura, R. Kuroiwa, H. Kano, and H. Kosaka, Optical holonomic single quantum gates with a geometric spin under a zero field, Nat. Photonics 11, 309 (2017).

[69] B. B. Zhou, P. C. Jerger, V. O. Shkolnikov, F. Joseph Heremans, G. Burkard, and D. D. Awschalom, Holonomic Quantum Control by Coherent Optical Excitation in Diamond, Phys. Rev. Lett. 119, 140503 (2017).

[70] D. Møller, L. B. Madsen, and K. Mølmer, Quantum Gates and Multiparticle Entanglement by Rydberg Excitation Blockade and Adiabatic Passage, Phys. Rev. Lett. 100, 170504 (2008).

[71] Y.-C. Zheng and T. A. Brun, Geometric manipulation of ensembles of atoms on an atom chip for quantum computation, Phys. Rev. A 86, 032323 (2012).

[72] I. I. Beterov, M. Saffman, E. A. Yakshina, V. P. Zhukov, D. B. Tretyakov, V. M. Entin, I. I. Ryabtsev, C. W. Mansell, C. MacCormick, S. Bergamini, and M. P. Fedoruk, Quantum gates in mesoscopic atomic ensembles based on adiabatic passage and Rydberg blockade, Phys. Rev. A 88, 010303(R) (2013).

[73] H. Wu, X.-R. Huang, C.-S. Hu, Z.-B. Yang, and S.-B. Zheng, Rydberg-interaction gates via adiabatic passage and phase control of driving fields, Phys. Rev. A 96, 022321 (2017).

[74] P. Z. Zhao, X. Wu, T. H. Xing, G. F. Xu, and D. M. Tong, Nonadiabatic holonomic quantum computation with Rydberg superatoms, Phys. Rev. A 98, 032313 (2018).

[75] Y.-H. Kang, Y.-H. Chen, Z.-C. Shi, B.-H. Huang, J. Song, and Y. Xia, Nonadiabatic holonomic quantum computation using Rydberg blockade, Phys. Rev. A 97, 042336 (2018).

[76] C.-P. Shen, J.-L. Wu, S.-L. Su, and E. Liang, Construction of robust Rydberg controlled-phase gates, Opt. Lett. 44, 2036 (2019).

[77] K.-Y. Liao, X.-H. Liu, Z. Li, and Y.-X. Du, Geometric Rydberg quantum gate with shortcuts to adiabaticity, Opt. Lett. 44, 4801 (2019).

[78] B.-J. Liu, Z.-Y. Xue, and M.-H. Yung, Brachistochronic nonadiabatic holonomic quantum control, arXiv:2001.05182.

[79] T. Chen, P. Shen, and Z.-Y. Xue, Robust and Fast Holonomic Quantum Gates with Encoding on Superconducting Circuits, Phys. Rev. Appl. 14, 034038 (2020).

[80] T. Chen and Z.-Y. Xue, Robust geometric quantum computation with time-optimal control, arXiv:2001.05789.

[81] A. Friedenauer and E. Sjöqvist, Noncyclic geometric quantum computation, Phys. Rev. A 67, 024303 (2003).

[82] Q.-X. Lv, Z.-T. Liang, H.-Z. Liu, J.-H. Liang, K.-Y. Liao, and Y.-X. Du, Noncyclic geometric quantum computation with shortcut to adiabaticity, Phys. Rev. A 101, 022330 (2020).

[83] Y.-Y. Jau, A. Hankin, T. Keating, I. Deutsch, and G. Biedermann, Entangling atomic spins with a Rydberg-dressed spin-flip blockade, Nat. Phys. 12, 71 (2016).

[84] D. Kult, J. Aberg, and E. Sjöqvist, Noncyclic geometric changes of quantum states, Phys. Rev. A 74, 022106 (2006).

[85] S.-L. Zhu and Z. D. Wang, Unconventional Geometric Quantum Computation, Phys. Rev. Lett. 91, 187902 (2003).

[86] G. Lindblad, On the generators of quantum dynamical semigroups, Commun. Math. Phys. 48, 119 (1976).

[87] S. B. Zheng, C. P. Yang, and F. Nori, Comparison of the sensitivity to systematic errors between nonadiabatic non-Abelian geometric gates and their dynamical counterparts, Phys. Rev. A 93, 032313 (2016).

[88] R. Barends, J. Kelly, A. Megrant, A. Veitia, D. Sank, E. Jeffrey, T. C. White, J. Mutus, A. G. Fowler, B. Campbell et al., 
Superconducting quantum circuits at the surface code threshold for fault tolerance, Nature (London) 508, 500 (2014).

[89] K. Singer, J. Stanojevic, M. Weidemüller, and R. Côté, Longrange interactions between alkali Rydberg atom pairs correlated to the ns-ns, np-np and nd-nd asymptotes, J. Phys. B: Atom. Mol. Opt. Phys. 38, S295 (2005); W. Li, D. Viscor, S. Hofferberth, and I. Lesanovsky, Electromagnetically Induced Transparency in an Entangled Medium, Phys. Rev. Lett. 112,
243601 (2014); D. Cano and J. Fortágh, Multiatom entanglement in cold Rydberg mixtures, Phys. Rev. A 89, 043413 (2014).

[90] M. A. Nielsen, A simple formula for the average gate fidelity of a quantum dynamical operation, Phys. Lett. A 303, 249 (2002).

[91] A. G. White, A. Gilchrist, G. J. Pryde, J. L. O'Brien, M. J. Bremner, and N. K. Langford, Measuring two-qubit gates, J. Opt. Soc. Am. B 24, 172 (2007). 